Bundesgesundheitsbl 2018 $61: 249-251$ https://doi.org/10.1007/s00103-018-2714-2 Online publiziert: 21. Februar 2018 (c) Springer-Verlag GmbH Deutschland, ein Teil von Springer Nature 2018

CrossMark

Wolfgang Lauer · Thomas Sudhop · Karl Broich

Bundesinstitut für Arzneimittel und Medizinprodukte, Bonn, Deutschland

\title{
E-Health und Medizinprodukte
}

\section{Bundesgesundheitsblatt Schwerpunktheft}

Sehr geehrte Leserinnen und Leser,

E-Health, Digitalisierung, GesundheitsApps, Wearables - diese Schlagworte sind aktuell in aller Munde. Je nach Sichtweise versprechen sie entweder unbegrenzte Möglichkeiten vernetzter „Gesundheitsversorgung 4.0" oder öffnen Tür und Tor für den Missbrauch persönlicher Gesundheitsinformationen.

Tatsache ist, dass aktuell ein grundlegender Wandel festzustellen ist. Es entstehen neue Kommunikationskanäle zwischen Patienten und Behandelnden, es eröffnen sich neue Möglichkeiten, eigene Gesundheitsdaten zu jedem Zeitpunkt einfach zu erfassen und auszuwerten. Nicht zuletzt wandeln sich Erwartungen an Prozesse, Inhalte, Verfügbarkeit und Transparenz der Gesundheitsversorgung.

Wesentlicher Treiber dieses Wandels ist der extrem dynamische Markt digitaler Gesundheitsanwendungen und -produkte von medizinischen Apps über Wearables bis hin zu Expertensystemen für Diagnose und Therapie. Entsprechende Produkte mit medizinischer Zweckbestimmung sind aus regulatorischer Sicht im Allgemeinen Medizinprodukte und unterliegen damit denselben gesetzlichen Regelungen wie z.B. Infusionspumpen, Computertomographen oder künstliche Herzklappen. Insbesondere bei digitalen Medizinprodukten, bei denen meist nicht mehr die direkte Interaktion mit dem Körper des Patienten im Vordergrund steht, sondern die Kritikalität einer generierten Information, wird es im Rahmen der neuen europäischen Medizinprodukteverordnung (MDR) zu deutlichen Verschärfungen dieser Regelungen kommen.

Das vorliegende Schwerpunktheft „EHealth und Medizinprodukte" beleuchtet die aktuellen Entwicklungen und Besonderheiten in diesem Themenbereich. Dabei wird das Thema aus verschiedenen Perspektiven betrachtet und diskutiert: klinisch, rechtlich, aus Sicht von Startups, regulatorisch/behördlich sowie abschließend mit einem Ausblick auf die mögliche Zukunft „Gesundheit 4.0“.

In drei Beiträgen aus der klinischen Perspektive wird zunächst die aktuelle Realität in der Klinik dargestellt. Was sehen wir? Was wird im Alltag genutzt? Welche Risiken bringt das mit sich? Welches Bewusstsein besteht für diese Problematik?

So systematisieren Gehring et al. zunächst den aktuellen Stand unterschiedlicher digitaler Unterstützungssysteme für klinisches Datenmanagement, therapeutische Prozesse und ärztliche Entscheidungen. Sie stellen dar, wie entsprechende Systeme unterschiedlicher Autonomiegrade Einzug in die klinische Anwendung gefunden haben, und diskutieren resultierende Herausforderungen u. a. für das ärztliche Handeln und die medizinische Ausbildung.

Der nachfolgende Beitrag von Lüttke et al. nimmt speziell die Diagnostik und Therapie psychischer Störungen in den Blick. Er stellt diesbezügliche technische Möglichkeiten und Angebote zur Verwendung digitaler Medien, wie z.B. Apps, therapeutische Onlineprogramme oder Wearables, dar und diskutiert auf Basis aktueller Studienergebnisse die Frage, inwieweit entsprechende digitale Angebote Eingang in die Versorgung psychisch kranker Menschen finden können bzw. sollten.

Entscheidungsunterstützungssysteme für die Arzneimitteltherapie sind in der klinischen Praxis bereits etabliert, um immer komplexere Entscheidungen auf immer umfangreicherer Datengrundlage 
möglichst zuverlässig und individualisiert treffen zu können. Haefeli und Seidling stellen in ihrem Beitrag dar, welche Voraussetzungen und Herausforderungen es für die Einbindung entsprechender Systeme in den klinischen Alltag gibt und welcher Forschungs- bzw. Entwicklungsbedarf z. B. angesichts der Weiterentwicklung der personalisierten Medizin besteht.

E-Health-Angebote und Medizinprodukte, die in diesem Zusammenhang genutzt werden, sind auch aus einer rechtlichen Perspektive zu betrachten. Dies erfolgt im zweiten Themenblock.

Dabei werden zunächst die aktuellen wie auch die zukünftigen medizinprodukterechtlichen Rahmenbedingungen für entsprechende Produkte im Europäischen Wirtschaftsraum und damit auch in Deutschland dargestellt. Der Beitrag von Lücker stellt z. B. Vorgaben für Produkte mit medizinischer Zweckbestimmung denen anderer digitaler Produkte im Gesundheitsbereich gegenüber und geht auf die resultierenden rechtlichen Anforderungen ein.

Auch wenn oder gerade weil Aspekte des Datenschutzes nicht unbedingt Bestandteil der medizinprodukterechtlichen Zertifizierung sind, ist der Gesundheitsdatenschutz angesichts der zunehmenden Vernetzung datenerfassender und -auswertender E-HealthProdukte von großer Wichtigkeit. Der Beitrag von Weichert sensibilisiert für entsprechende Risiken, erläutert maßgebliche Datenschutzprinzipien bzw. gesetzliche Regelungen und benennt weiteren Handlungsbedarf.

In Gesprächen des Bundesinstituts für Arzneimittel und Medizinprodukte (BfArM) mit Start-ups aus dem E-Health-Sektor zeigt sich immer wieder, dass der Weg vom erkannten Bedarf und der technischen Lösungsidee über die CE-Zertifizierung als Medizinprodukt bis hin zur meist angestrebten Erstattung durch die gesetzliche Krankenversicherung als sehr lang, aufwendig und aus Start-up-Sicht fremd und komplex empfunden wird. Um hier ein besseres Gesamtverständnis für die erforderlichen Entscheidungen, Anforderungen und resultierenden Vorbereitungen $\mathrm{zu}$ schaffen, wird im dritten Abschnitt der
Weg von der Idee bis zur Erstattung über alle Wegstrecken erläutert.

Im Beitrag von Hagen und Lauer geht es zunächst um die Herausforderungen und Unterstützungsbedarfe, die seitens Start-ups gesehen werden. Dazu werden die Ergebnisse einer Onlineumfrage unter entsprechenden Unternehmen sowie Forschungsgruppen vorgestellt.

Ergänzend stellen im nächsten Beitrag zwei Start-ups anhand unterschiedlicher digitaler Medizinprodukte ihre Erfahrungen auf dem zurückgelegten Weg vor. Herberz et al. erläutern dabei sowohl positive Auswirkungen der Medizinproduktezertifizierung, z. B. für die bessere Strukturierung ihrer Entwicklungsprojekte, als auch wahrgenommene Hürden, insbesondere im Rahmen des Zugangs zur Kostenerstattung.

Ausschlaggebend für den Weg des Marktzugangs und u.a. die Frage, ob ein E-Health-Produkt ein Medizinprodukt ist, ist die Zweckbestimmung des Herstellers. Das BfArM bietet Startups und Forschungsgruppen vielfältige Unterstützung bei diesen wichtigen Entscheidungen. Rämsch et al. erläutern in ihrem Beitrag diese Angebote und stellen insbesondere die Orientierungshilfe des BfArM zur Abgrenzung und Risikoklassifizierung bei Medical Apps vor.

Je nach Risikoklasse muss die Konformitätsbewertung als Medizinprodukt unter Einbeziehung einer sogenannten Benannten Stelle erfolgen, die diesbezügliche Sicherheits- und Leistungsnachweise überprüft. Berensmann und Gratzfeld erläutern in ihrem Beitrag ausführlich die Anforderungen, die von Benannten Stellen an entsprechende Produkte und damit auch an die Hersteller gestellt werden.

Medizinprodukte sowie auch ihre Hersteller und Betreiber unterliegen in Deutschland der Überwachung durch die Landesbehörden. Der Beitrag von Terhechte gibt einen Überblick über die diesbezüglichen Überwachungsaufgaben und -kriterien der Landesbehörden und richtet dabei den Blick u.a. auf das IT-Sicherheitsgesetz sowie auf die Vorgaben der neuen europäischen Medizinprodukteverordnung (MDR).

Als zentraler Baustein der Finanzierung von E-Health-Angeboten und gleichzeitig zeit- und kostenaufwendige Hürde wird meist der Zugang zur Erstattung durch die gesetzliche Krankenversicherung angesehen. In ihrem Beitrag erläutert Gregor-Haack die wichtigsten Finanzierungswege und Erstattungskategorien sowie bestehende Herausforderungen herkömmlicher Erstattungswege aufgrund der Neuartigkeit digitaler Angebote.

Der abschließende Themenblock widmet sich einem Blick in die Zukunft digitaler Gesundheitsangebote bzw. Medizinprodukte.

Unter dem Titel „Gesundheit 4.0 wie geht's uns denn morgen“ stellen Müschenich und Wamprecht mögliche Antworten auf die Frage nach dem Einfluss digitaler Medizin auf die zukünftige $\mathrm{Ge}$ sundheitsversorgung vor. Sie identifizieren diesbezügliche zentrale Technologien bzw. Anwendungsbereiche und skizzieren erwartete Auswirkungen u. a. auf die zukünftige Arzt-Patienten-Kommunikation.

Albrecht et al. diskutieren Herausforderungen an die Nutzenbewertung digitaler Gesundheitsprodukte. Sie stellen dar, inwiefern sich entsprechende Produkte von klassischen Medizinprodukten und Arzneimitteln unterscheiden, welche Konsequenzen dies im Hinblick auf die derzeit angewandten Methoden zur Nutzenbewertung hat und welche Weiterentwicklung diese aus ihrer Sicht erfahren sollten.

Bei aller Faszination neuer technischer wie medizinischer Möglichkeiten und gesundheitsökonomischer Effizienzpotenziale darf nicht vergessen werden, dass die zunehmende Entwicklung und Nutzung digitaler Gesundheitsangebote fundamentalen Einfluss auf Patienten sowie auf Behandelnde hat. Mit der Veränderung von Zugangswegen zur Versorgung, des Arzt-PatientenVerhältnisses sowie zunehmender Datengenerierung und -auswertung stellen sich auch viele medizinethische Fragen. Der abschließende Beitrag von Groß und Schmidt widmet sich daher dem wichtigen Feld der ethischen Bewertung von E-Health und der Rückwirkungen der Digitalisierung des Gesundheitsmarktes auf Nutzer bzw. Patienten und Behandelnde. 
Liebe Leserinnen und Leser, wir als Editoren dieses Schwerpunktheftes freuen uns, dass die verschiedenen Beiträge sowohl einen Überblick über ganz unterschiedliche Aspekte des hochaktuellen und sehr dynamischen Themas E-Health und Medizinprodukte geben als auch konkrete Problemstellungen und Lösungsansätze im Detail darstellen. Ihnen wünschen wir daher eine interessante und anregende Lektüre.

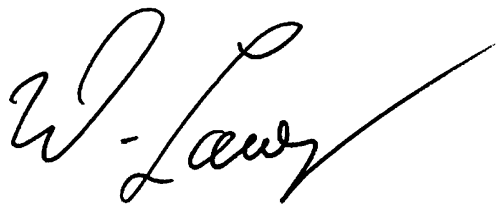

Dr. Wolfgang Lauer,

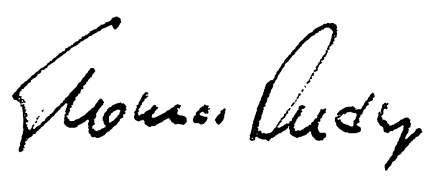

PD Dr. Thomas Sudhop

und

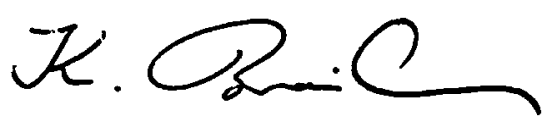

Prof. Dr. Karl Broich

\section{Korrespondenzadresse}

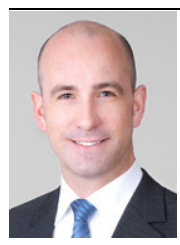

Dr. W. Lauer

Bundesinstitut für

Arzneimittel und

Medizinprodukte

Kurt-Georg-Kiesinger-Allee 3,

53175 Bonn, Deutschland

Wolfgang.Lauer@bfarm.de

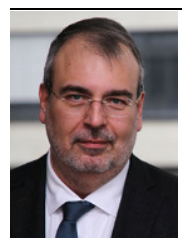

PD Dr. T. Sudhop

Bundesinstitut für

Arzneimittel und

Medizinprodukte

Kurt-Georg-Kiesinger-Allee 3,

53175 Bonn, Deutschland

thomas.sudhop@bfarm.de

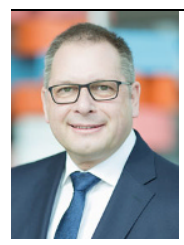

Prof. Dr. K. Broich

Bundesinstitut für

Arzneimittel und

Medizinprodukte

Kurt-Georg-Kiesinger-Allee 3,

53175 Bonn, Deutschland

leitung@bfarm.de

Interessenkonflikt. W. Lauer, T. Sudhop und K. Broich geben an, dass kein Interessenkonflikt besteht. 